\title{
On the $\mathrm{Br} \gamma$-line emission of the Herbig Ae/Be star MWC 120
}

\author{
Alexander Kreplin ${ }^{1 \star}$, Larisa Tambovtseva ${ }^{2}$, Vladimir Grinin ${ }^{2,3}$, Stefan Kraus ${ }^{1}$, \\ Gerd Weigelt ${ }^{4}$, Yang Wang 4 \\ ${ }^{1}$ University of Exeter, Astrophysics Group, Stocker Road, Exeter, EX4 4QL, UK \\ ${ }^{2}$ Pulkovo Observatory of RAS, Pulkovskoe shosse 65, St. Petersburg 196140, Russia \\ ${ }^{3}$ The V.V. Sobolev Astronomical Institute of the St. Petersburg University, Petrodvorets, St. Petersburg 198904, Russia \\ ${ }^{4}$ Max Planck Institut für Radioastronomie, Auf dem Hügel 69, 53121 Bonn, Germany
}

Accepted XXX. Received YYY; in original form ZZZ

\begin{abstract}
The origin of the $\mathrm{Br} \gamma$ line in Herbig Ae/Be stars is still an open question. It has been proposed that a fraction of the $2.166 \mu \mathrm{m} \mathrm{Br} \gamma$ emission might emerge from a disc wind, the magnetosphere, and other regions. Investigations of the $\operatorname{Br} \gamma$ line in young stellar objects are important to improve our understanding of the accretion-ejection process. Near-infrared long-baseline interferometry enables the investigation of the $\mathrm{Br} \gamma$ line emitting region with high spatial and high spectral resolution. We observed the Herbig Ae/Be star MWC 120 with the VLTI/AMBER instrument in different spectral channels across the $\operatorname{Br} \gamma$ line with a spectral resolution of $R \sim 1500$. Comparison of the visibilities, differential, and closure phases in the continuum and the line-emitting region with geometric and radiative transfer disc-wind models lead to constraints on the origin and dynamics of the gas emitting the $\mathrm{Br} \gamma$ light. Geometric modelling of the visibilities reveals a line emission region about two times smaller than the $K$-band continuum region, which indicates a scenario where the $\mathrm{Br} \gamma$ emission is dominated by an extended disc wind rather than by a much more compact magnetospheric origin. To compare our data to a physical model, we applied a state-of-the-art radiative transfer disc-wind model. We find that all measured visibilities, differential and closure phases of MWC 120 can approximately be reproduced by a disc-wind model. A comparison with other Herbig stars indicates a correlation of the modelled inner disc-wind radii with the corresponding Alfvén radii for late spectral type stars.
\end{abstract}

Key words: circumstellar matter - stars: individual: MWC 120 - stars: formation stars: pre-main-sequence - stars: emission-line, Be - techniques: interferometric

\section{INTRODUCTION}

Studying the accretion and outflow processes in young stellar objects (YSOs) is of fundamental importance to improve our understanding of star and planet formation. During the star formation process, the infalling gas and dust form a circumstellar disc around the protostellar object. These discs are believed to be the birthplace of planets. During the collapse of the parental molecular cloud, the initial matter is redistributed (1) through accretion inward onto the central protostellar object and (2) outward in the form of outflows. Most of the circumstellar disc mass consists of gas, in particular hydrogen, that can be traced by spectral lines. The line profiles and their place of origin provide crucial infor-

^ E-mail: akreplin@astro.ex.ac.uk mation for constraining different inflow and outflow scenarios (e.g., disc wind, magnetospheric accretion). An important tracer is the $\operatorname{Br} \gamma 2.166 \mu \mathrm{m}$ line, which can also be used to estimate the mass accretion rate (Muzerolle et al. 1998; Garcia Lopez et al. 2006). The scientific merit of spectrally and spatially resolved $\operatorname{Br} \gamma$ line observations of Herbig $\mathrm{Ae} / \mathrm{Be}$ stars and its comparison to disc-wind and magnetosphere models was shown in literature (e.g. Weigelt et al. 2011; Caratti o Garatti et al. 2015; Garcia Lopez et al. 2015; Kurosawa et al. 2016). In this work, we expand the sparse sample of available detailed interferometric studies on the $\mathrm{Br} \gamma$ line emission region in Herbig stars by presenting new observations of the Herbig Ae/Be star MWC 120. The growing sample of detailed studies of Herbig stars of different spectral types, different luminosities and inclinations of the disc axies relative to the sky plane enables the systematic 
Table 1. Observation log of the VLTI/AMBER medium-resolution $(R \sim 1500)$ observations of MWC 120 conducted with the UT2-UT3UT4 configuration.

\begin{tabular}{ccccccc}
\hline UT Date & $\begin{array}{c}\text { UT Time } \\
{[\mathrm{h}: \mathrm{m}]}\end{array}$ & $\begin{array}{c}\text { DIT } \\
{[\mathrm{ms}]}\end{array}$ & $\begin{array}{c}\text { NDIT } \\
\#\end{array}$ & $\begin{array}{c}\text { Proj. baselines } \\
{[\mathrm{m}]}\end{array}$ & $\begin{array}{c}\text { PA } \\
{[\circ]}\end{array}$ & Calibrator \\
\hline $2012-11-04$ & $06: 53-07: 00$ & 500 & 600 & $43.40 / 62.46 / 87.89$ & $39.90 /-71.08 /-98.53$ & HIP 26061 \\
$2012-12-29$ & $04: 44-04: 51$ & 500 & 600 & $46.32 / 58.27 / 87.77$ & $45.03 /-68.57 /-97.49$ & HIP 27 980 \\
$2012-12-30$ & $05: 13-05: 21$ & 500 & 600 & $45.63 / 54.64 / 84.30$ & $45.81 /-66.64 /-97.39$ & HD 33208 \\
$2013-02-28$ & $01: 17-01: 24$ & 500 & 600 & $46.63 / 54.70 / 84.37$ & $45.81 /-66.67 /-97.39$ & HD 33 208 \\
\hline
\end{tabular}

investigation of the physical origin of the disc wind emission region.

MWC 120 (HD 37806) is a Herbig late Be or early Ae type object (van den Ancker et al. 1998; de Winter et al. 2001; Wade et al. 2007) located at a distance of about 375 pc (Alecian et al. 2013). The emission line characteristic was first reported by Merrill \& Burwell (1933). The stellar mass ranges between $\sim 3-4 M_{\odot}$ (Mariñas et al. 2011; Cauley \& Johns-Krull 2015). Spectropolarimetric measurements show an intrisic linear polarization angle between 115-138 (Mottram et al. 2007; Oudmaijer \& Drew 1999; Yudin 2000). Furthermore, MWC 120 shows $\mathrm{H} \alpha$ P Cygni profiles with strong variability of the blueshifted absorption component Harrington \& Kuhn (2007) indicating an outflowing wind structure. $H$-band interferometric measurements revealed a circumstellar disc around MWC 120 seen under an inclination of $\sim 41.4^{\circ}$ with a position angle (PA) of the system axis of $\sim 135^{\circ}$ (North is up, East to the left). The continuum emission in the $H$ - and $K$-band predominately emerge from a region within radius of $\sim 1.6$ mas (Lazareff et al. 2017; Eisner et al. 2004) corrsponding to 0.6 au at a distance of 375 pc.

In this paper, we present the first near-infrared interferometric observations of the $K$-band continuum and the $\mathrm{Br} \gamma$ line-emitting region of MWC 120 with a spectral resolution of $R \sim 1500$. In Section 2, we describe our observations and the data reduction. In Section 3, we discuss our geometric and radiative transfer modelling. We present the results in Section 4, the discussion in Section 5, and give a summary in Section 6.

\section{OBSERVATIONS AND DATA REDUCTION}

The Herbig Ae/Be star MWC 120 was observed with the near-infrared VLTI/AMBER interferometer in four nights in December 2012 and in February 2013 (see Table 1). The AMBER instrument (Petrov et al. 2007) creates spectrally dispersed interferograms by combining the light of three telecopes. The observations were conducted in service mode with the medium spectral resolution $(\mathrm{MR})$ mode $(R \sim 1200)$ centred at the $\operatorname{Br} \gamma$ line. The exposure time per frame was $500 \mathrm{~ms}$ using the fringe tracker FINITO to stabilize the fringes. The tracking performance of FINITO can be different for target and calibrator observations and therefore might introduce a systematic bias in the continuum visibilites. Therefore, we use low resolution (LR) AMBER data (IDs: 084.C-0187, 086.C-0267) recorded without the fringe tracker to calibrate the MR continnum visibilites. Due to the relative low number of available LR $u v$-points, we cal- ibrated the continuum visibilities at the available $12 \mathrm{MR}$ $u v$-positions where we assume the inclination and PA of the MWC 120 system from literature of $\sim 41^{\circ}$ and $\sim 135^{\circ}$, respectively (Lazareff et al. 2017). The data reduction was performed using the data reduction software amdlib v3.0.5 ${ }^{1}$. The frame selection was set to 20 per cent of the highest fringe SNR (see e.g. Tatulli et al. 2007). The observation log is shown in Table 1.

\section{MODELLING}

\subsection{Geometric models}

For the characterization of the near-infrared continuum- and line- emitting region, we first fit geometric models (Gaussian distribution, ring) and a temperature gradient model (TGM) to the observed visibilities (see Fig. 1 a, data points in second row). We first fitted the $K$-band continuum visibilites with a temperature gradient model using a fixed elongation of 1:1.3 (minor to major axis), corresponding to the literature value of the system inclination of $\sim 41^{\circ}$ (Lazareff et al. 2017).

Our model visibilities incorporate the central stellar emission, the circumstellar $K$-band continuum, and the line emitting region. The total visibility can be written as

$$
V_{\text {tot }}=\frac{\left|F_{\star} V_{\star}+F_{\text {cont }} V_{\text {cont }}+F_{\text {line }} V_{\text {line }}\right|}{F_{\star}+F_{\text {cont }}+F_{\text {line }}}
$$

where $F_{\star}$ denotes the stellar flux, $F_{\text {cont }}$ the continuum flux component, and $F_{\text {line }}$ the flux of the $\mathrm{Br} \gamma$-line component. $V_{\star}, V_{\text {cont }}$ and $V_{\text {line }}$ denote the complex visibility of the star, the $K$-band continuum and the line-emitting component, respectively. The central star is unresolved by our measurements and therefore we set $V_{\star}=1$ (the stellar radius is about 0.07 mas at $D=375 \mathrm{pc}$ ). The stellar component of the dereddened SED of MWC 120 was fitted by a Kurucz model (Kurucz 1979). We then estimated the circumstellar-to-stellar flux ratio by calculating the difference of the observed flux to the Kurucz spectrum for $\lambda=2.2 \mu \mathrm{m}\left(T_{\star}=10000 \mathrm{~K}\right.$, $\log (g)=4.0$; Alecian et al. 2013). We derived a circumstellarto-stellar flux ratio of $f_{K}=F_{\text {circ }} / F_{\star}=6.7$ (with a typical error of 10-20 per cent).

The obtained best fit of a TGM model, describing the continuum and line emission, TGM-TGM (model A), is presented in Appendix A1. The temperature gradient model is described by an inner radius $R_{\mathrm{in}}$, an outer radius $R_{\text {out }}$ and the temperature profile $T(R)=T_{d}\left(R_{\text {in }}\right)\left(R / R_{\text {in }}\right)^{-q}$, where

1 The AMBER reduction package amdlib is available at: http://www.jmmc.fr/data_processing_amber.htm 

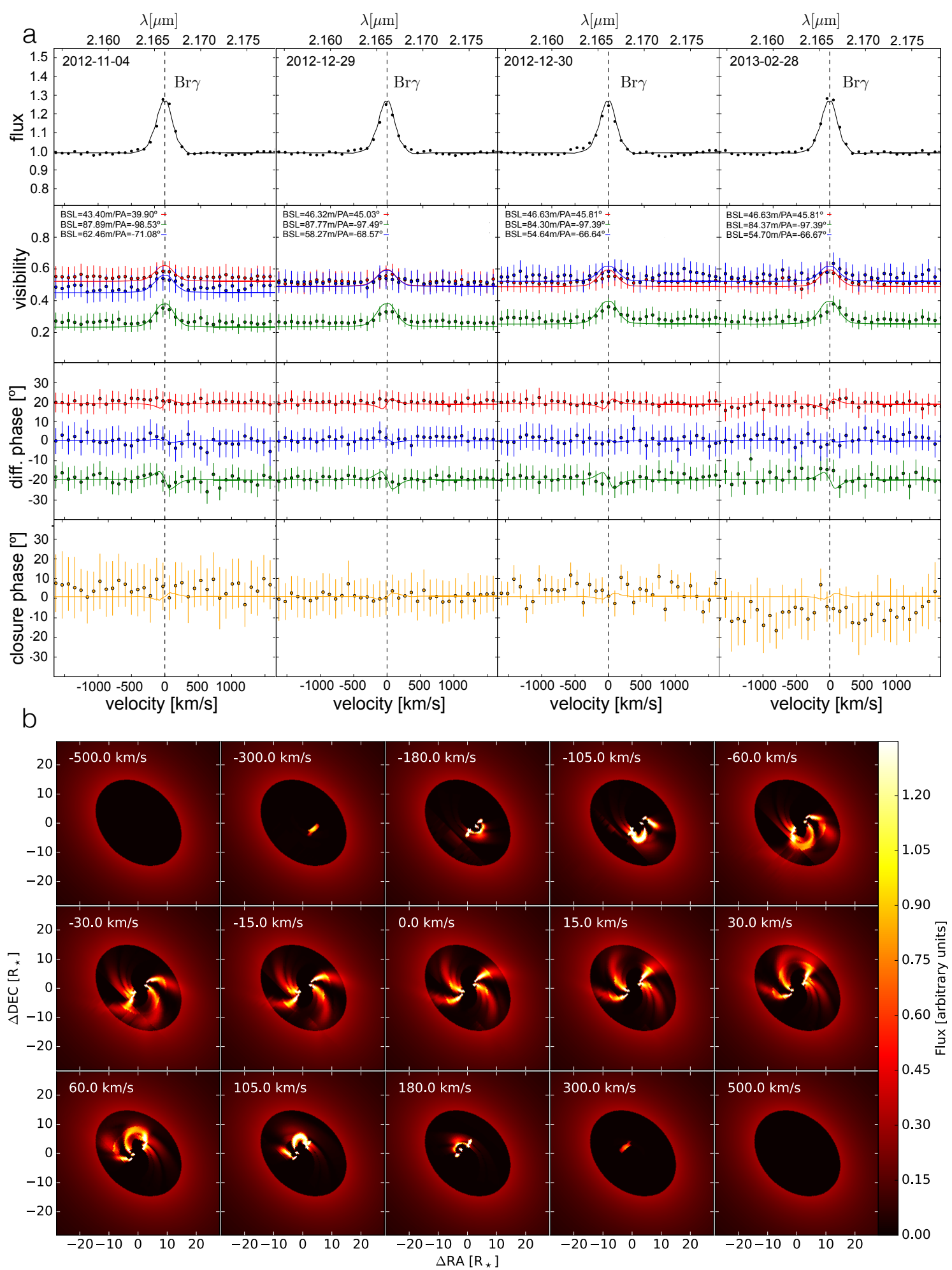

Figure 1. a: Comparison of the MWC 120 measurements (data points) with the best-fitting disc-wind model of the four different obervations (left to right). Top panels: Average calibrated spectrum of MWC 120 taken with three UT telescopes during the interferometric observation. The black line represents the model spectrum of the disc-wind model. Middle panels: Visibilities of the three different baselines (red, green, blue) on the upper row and the corresponding differential phases (bottom row). Bottom: Closure phases of each baseline triplet. For clarity, we indicated the position of the $\operatorname{Br} \gamma 2.1661 \mu \mathrm{m}$ line by a vertical dashed line in all panels. b: Channel maps of the best-fitting disc-wind model intensity distribution (square-root scaling) ranging from $-300 \mathrm{~km} \mathrm{~s}^{-1}$ (upper left) to $300 \mathrm{~km}$ $\mathrm{s}^{-1}$ (lower right). The star is omitted in this representation. The different model components are clearly visible, 1) the outer continuum disc structure that is modelled by a temperature gradient disc (best visible without any line emission at the velocity channels outside the emission line at $\sim 500 \mathrm{~km} \mathrm{~s}^{-1}$ and 2) the inner disc-wind structure dominating the emission within the Br $\gamma$ line (visible across the channels $-300 \mathrm{~km} \mathrm{~s}^{-1}$ to $+300 \mathrm{~km} \mathrm{~s}^{-1}$ ). Please note the shift of the photoscentre from the lower right to the upper left that is required to successfully fit the measured differential phases (see upper panel $a$, third row). 


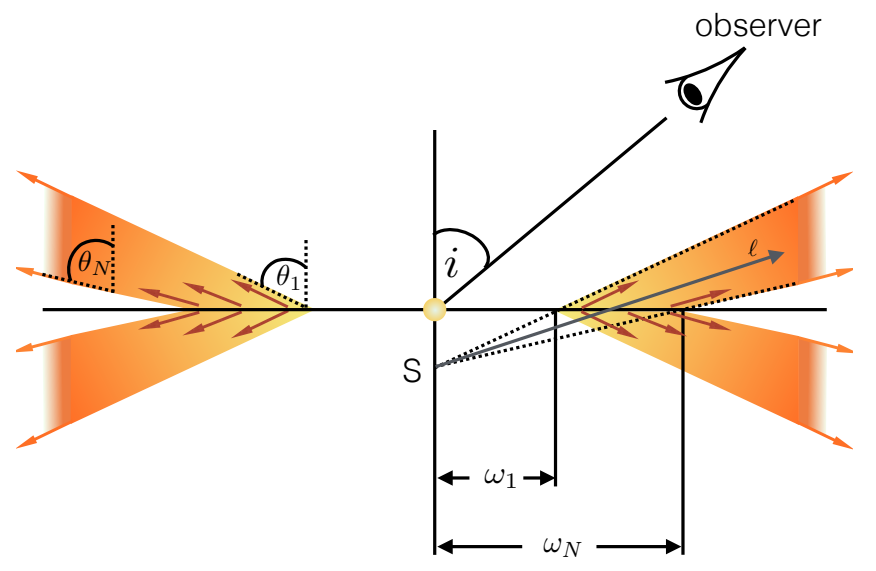

Figure 2. Sketch of the disc-wind geometry assumed in the presented calculation. The whole scene is seen by an observer under the inclination $i$.

Table 2. Best-fitting parameters of the continuum and line visibility fits.

\begin{tabular}{llcccc}
\hline Model & \multicolumn{2}{c}{ components } & $\begin{array}{c}R_{\text {in }} \\
{[\mathrm{mas}]}\end{array}$ & $\begin{array}{c}\text { HWHM } \\
{[\mathrm{mas}]}\end{array}$ & $\chi_{\text {red }}^{2}$ \\
\hline \multirow{2}{*}{$\mathrm{A}$} & continum & TGM & $0.94 \pm 0.12$ & - & 0.58 \\
& line & TGM & & $0.41 \pm 0.13$ & \\
\hline \multirow{2}{*}{$\mathrm{B}$} & continum & Gauss & & $2.25 \pm 0.14$ & \multirow{2}{*}{1.89} \\
& line & Gauss & & $1.26 \pm 0.11$ & \\
\hline \multirow{2}{*}{$\mathrm{C}$} & continum & ring & $1.78 \pm 0.20$ & & \multirow{2}{*}{11.37} \\
& line & ring & $1.20 \pm 0.12$ & & \\
\hline
\end{tabular}

$T_{d}$ denotes the temperature at $R_{\text {in }}$ (for details see, e.g., Kreplin et al. 2012). For the parameter $q$, our fit yields a value of 0.4 and the outer radius was kept at the value of the field-of-view of our UT observation of 60 mas. In addition, we calculated a Gauss-Gauss distribution (model $\mathrm{B}$ ) and a ring-ring distribution (model $\mathrm{C}$, see Table 2). The average size ratio between the $K$-band continuum and the line emitting region is $\sim 1.9$. We derived a $K$-band inner disc radius in the continuum of $0.94 \pm 0.12$ mas (corresponding to $0.35 \pm 0.05$ au at a distance of $375 \mathrm{pc}$ ). The $\mathrm{Br} \gamma$ line emitting region is about two times more compact than the continuum region and has an inner model disc radius of $0.41 \pm 0.13$ mas (corresponding to $0.15 \pm 0.05$ $\mathrm{au}$ at a distance of $375 \mathrm{pc}$ ). For error calculation, we used bootstrapping (over baselines) to create 1000 artifical data sets that were used again for fitting. An individual set of new best-fitting parameters was derived for each fit. The spread of the parameter values can be represented by a histograms derived from the best-fitting values using the 1000 randomly created new data sets. A $1 \sigma$-Gauss fit to the histogram of each fit parameter was used to estimate the errors. All best-fitting parameters are summarized in Table 2. The continnum model used for further analysis consists of a temperature-gradient $\operatorname{disc}\left(T_{d}=1500 \mathrm{~K}\right.$, temperature exponent $\mathrm{q}=0.4)$ starting at $\sim 17$ stellar radii $(\sim 0.35$ au at a distance of $375 \mathrm{pc}$ ) which is consistent with the theoretical dust sublimation radius $\left(T_{\text {dust }}=1500 \mathrm{~K}\right)$ of 0.347 au if we assume the stellar luminosity of $L_{\star} \sim 100 L_{\odot}$ determined by
Vinković \& Jurkić (2007).

\subsection{Disc-wind model}

Because of the centro-symmetric nature of the temperaturegradient model brightness distribution, it cannot be used to model non-zero differential and closure phases of our data. Therefore, we apply a physical disc-wind model. We calculated disc-wind models and compared the observed line profiles, visibilities, differential, and closure phases to the corresponding line profiles, visibilities, and phases of the disc-wind model. In the following we summarize the most important model properties (for more details on the discwind model please see Tambovtseva et al. 2014).

The complete model consists of a temperature-gradient model representing the continuum emission and the radiative transfer disc-wind model describing the $\mathrm{Br} \gamma$ emission. The applied disc-wind model is based on the magnetocentrifugal disc wind (Blandford \& Payne 1982) and was developed for Herbig Ae/Be stars, described in detail in Grinin \& Tambovtseva (2011) and Weigelt et al. (2011).

The basic input parameters are the inner and outer halfopening angle $\theta_{1}$ and $\theta_{N}$ of the disc wind, the inner and outer radius of the disc-wind base $\omega_{1}$ and $\omega_{N}$, the mass load parameter $\gamma$, the acceleration parameter $\beta$, the mass loss rate $\dot{M}$, and inclination and PA of the disc $i$ and $P A$, respectively. Fig. 2 shows a sketch of the disc-wind geometry. Based on the centre of the coordinates $S$, the tangential velocity component $u(\omega)$ and the poloidal velocity component $v(\ell)$ along the streamlines are given by:

$$
u(\omega)=u_{K}\left(\omega_{i}\right)\left(\omega / \omega_{i}\right)^{-1} \text { and } v(\ell)=v_{0}+\left(v_{\infty}-v_{0}\right)\left(1-\ell_{i} / \ell\right)^{\beta},
$$

where $u_{K}$ denotes the Keplerian velocity in the i-th footpoint, $\omega_{i}$ the cylindrical radius at the footpoint of the i-th streamline, $v_{0}$ an initial poloidal velocity (assumed equal to the thermal velocity), $v_{\infty}$ the terminal velocity, and $\ell_{i}$ the distance from the point $S$ to the footpoint of the i-th streamline.

In our model, we use for simplicity a disc wind with a constant temperature $(10000 \mathrm{~K})$ that consists only of hydrogen atoms. This approximation refers to the class of "warm discwind" models where the disc wind of young stars can be heated up to the temperature of about $10^{4} \mathrm{~K}$ by ambipolar diffusion, i.e. a drift of the charged particles relative to neutrals (Safier 1993; Garcia et al. 2001). The excitation and ionization states have been calculated with the help of numerical codes developed by Grinin \& Katysheva 1980; Grinin \& Mitskevich 1990; Tambovtseva et al. 2001, optimized for media with large velocity gradients. Transitions between the discrete levels within the equations of statistical equilibrium are calculated in the Sobolev approximation (Sobolev 1960). The gas temperature rapidly reaches $10^{4} \mathrm{~K}$ with increasing height from the disc plane but not near the disc surface. In these models, the wind electron temperature in the acceleration zone near the disc surface is not high enough to excite the $\mathrm{Br} \gamma$ line emission. Therefore, in our model, the lowtemperature region below a certain height above the disc plane does not contribute to the $\mathrm{Br} \gamma$ disc-wind emission. 
Table 3. Explored parameter space of the disc-wind model

\begin{tabular}{lcl}
\hline Description & Parameter & Range \\
\hline Inner half opening angle & $\theta_{1}$ & $30-45^{\circ}$ \\
Outer half opening angle & $\theta_{N}$ & $60-80^{\circ}$ \\
Inner radius & $\omega_{1}$ & $2-5 R_{\star}$ \\
Outer radius & $\omega_{N}$ & $6-10 R_{\star}$ \\
Mass load parameter & $\gamma$ & 3 \\
Acceleration parameter & $\beta$ & $3-5$ \\
Mass loss rate & $\dot{M}$ & $(0.5-2.0) 10^{-7} M_{\odot} \mathrm{yr}^{-1}$ \\
Inclination & $i$ & $30^{\circ}, 40^{\circ}, 50^{\circ}$ \\
System axis position angle & $\mathrm{PA}$ & $110^{\circ}-150^{\circ}$ \\
\hline
\end{tabular}

Table 4. Best model parameters of the disc wind

\begin{tabular}{ccccccccc}
\hline $\begin{array}{c}\theta_{1} \\
{\left[{ }^{\circ}\right]}\end{array}$ & $\begin{array}{c}\theta_{N} \\
{\left[{ }^{\circ}\right]}\end{array}$ & $\begin{array}{c}\omega_{1} \\
{\left[R_{\star}\right]}\end{array}$ & $\begin{array}{c}\omega_{N} \\
{\left[R_{\star}\right]}\end{array}$ & $\gamma$ & $\beta$ & $\begin{array}{c}\dot{M} \\
{\left[M_{\odot} \mathrm{yr}^{-1}\right]}\end{array}$ & $\begin{array}{c}i \\
{\left[{ }^{\circ}\right]}\end{array}$ & $\begin{array}{c}\mathrm{PA} \\
{\left[{ }^{\circ}\right]}\end{array}$ \\
\hline 45 & 80 & 2 & 10 & 3 & 5 & $10^{-7}$ & 40 & 135 \\
\hline
\end{tabular}

\section{RESULTS}

To get a first estimate of the ratio between the sizes of the $K$-band continuum emitting region and the region of $\mathrm{Br} \gamma$ emission of MWC 120, we fitted a TGM-disc to the AMBER visibilities. We derived an inner disc radius of the continuum emission of $0.94 \pm 0.12$ mas (corresponding to $0.35 \pm 0.05$ au at a distance of $375 \mathrm{pc}$ ). With a fitted inner radius of $0.41 \pm 0.13$ mas (corresponding to $0.15 \pm 0.05$ au at a distance of $375 \mathrm{pc}$ ), the $\mathrm{B} \gamma$-line emitting region is $\sim 2$ times more compact than the continuum emission.

In order to be able to reproduce all interferometric observable (visibilities, differential, and closure phases), we calculated about one hundred disc-wind models to explore the parameter space listed in Table 3. The following interative process has been applied. 1) We searched for a disc-wind model that is able to reproduce the spectral line profile and 2) calculated intensity maps in many velocity channels across the Br $\gamma$ line (from $-500 \mathrm{~km} \mathrm{~s}^{-1}$ to $+500 \mathrm{~km}^{-1}$; see Fig. $1 \mathrm{~b}$ ). From these intensity maps, we 3 ) extract the wavelengthdependent interferometric quantities that we compared to the observations until we find a good match. In Fig. 1a we show the comparison of the observed interferometric quantitites to our final disc-wind model. The best model parameters are summarized in Table 4. Due to the long computing times of radiative-transfer disc-wind models, an estimation of individual error bars on the model parameters is out of the scope of such an analysis. But because of the nature of the presented interferometric observations, we have both spectral and spatial information on the gas simultaneously. This reduces the ambiguities within our models. While different disc-wind models can reproduce the line profile equally good, the additional information from visibilities and differential and closure phases restricts the range of possible parameter values (seen in Table 3 ). While the inner disc-wind radius is mainly constrained by the visibilities, the gas kinematic is restriced by the spectral line profile and the differential and closure phases (e.g. derivation of direction of wind rotation). For some guidance about the quality of the model, we give a few $\chi_{\text {red }}^{2}$ values. Our best model shows a $\chi_{\text {red }}^{2}=1.06$. The

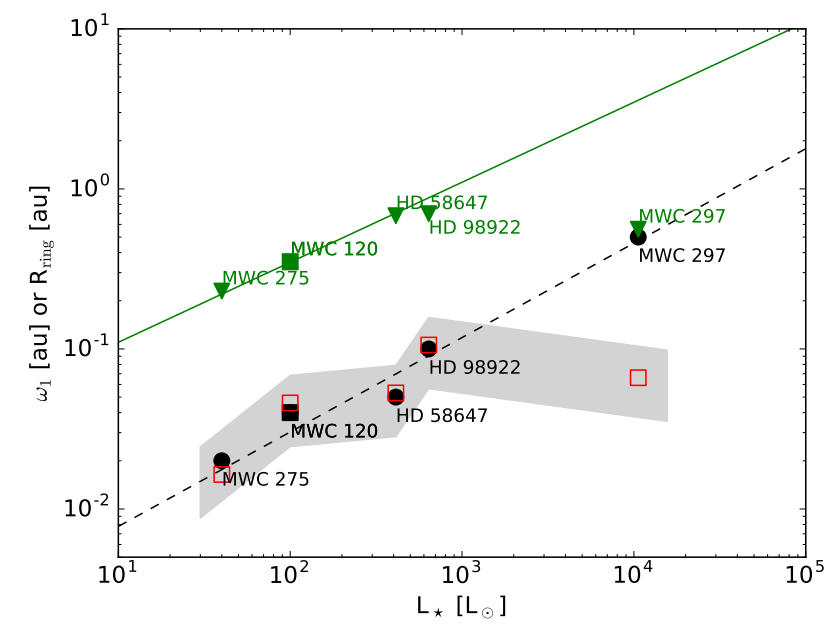

Figure 3. Size-luminosity relation showing the Herbig Ae/Be target sample of Kurosawa et al. 2016 (data points) overplotted with the new observation of MWC 120 . The continuum $K$-band ring fit radii are plotted as green triangles and the inner radii of the disc-wind base $\omega_{1}$ are plotted as solid black circles. Our new observation of MWC 120 is indicated by solid squares. The solid green line represents the expected dust sublimation radius (Monnier et al. 2005) for a dust sublimation temperature of $1500 \mathrm{~K}$. The dashed black line is a power law found to roughly describe the dependance of the disc-wind inner radii on the stellar luminosity (Kurosawa et al. 2016). The inner radii of the disc-wind base of the lower luminosity objects (MWC 275, MWC 120, HD58647, HD98922) can be approximately described by the corresponding Alfvén radii (red open squares, see text). The grey shaded area indicates the Alfvén radii of surface magnetic fields between 25150G, typical for Herbig Ae/Be stars (Wade et al. 2007). For the high luminosity target MWC 297, the derived inner disc-wind radius is $\sim 10$ times more extended than the calculated Alfvén radius.

most robust estimated model parameters are the inner radius and the inclination and position angle. An increase of the inner radius by $2 R_{\star}$ or larger leads to $\chi_{\text {red }}^{2}$ values greater than 2.56. The change of the inclination angle by $10^{\circ}$ leads to $\chi_{\text {red }}^{2}>2.38$, whereas a position angle difference from the best model of more than $30^{\circ}$ yields $\chi_{\text {red }}^{2}>2.42$.

As shown in Fig. 1, our final disc-wind model is able to approximately reproduce the spectral line shape (upper row in Fig. 1 a), the visibilities (second row), and the differential and closure phases (third and bottom row, respectively). The increase of the visibilities within the $\operatorname{Br} \gamma$ line require an emission line region more compact than the $K$-band continuum structure, as predicted by geometric modelling in Sec. 3.1. The base of the inner disc-wind radius $\omega_{1}$ is located at $\sim 2 R_{\star}$ (corresponding to 0.04 au at a distance of $375 \mathrm{pc}$ ).

\section{DISCUSSION}

The Br $\gamma$-line emission might emerge from different circumstellar regions dependent on the dominant mechanism including mass infall and outflow scenarios. Empirically, $\mathrm{Br} \gamma$ emission is correlated with the mass acccretion rate (Muzerolle et al. 1998) and might trace circumstellar gas in the magnetosphere close to the central star (van den Ancker 
2005). Contribution from a gaseous inner disc may also play a role (Muzerolle et al. 2004) although the fractional contribution to the total line emission is probably weak (Tambovtseva et al. 2016). An important emission process is the magnetocentrifugally driven disc wind where the hydrogen line emission can emerge from a more extended circumstellar region (Blandford \& Payne 1982).

Our geometric modelling indicates that the $\mathrm{Br} \gamma$ emission (emerging from a radius of $\sim 0.15 \mathrm{au}$ ) is about two times more compact than the continuum dust emission (at a radius of $\sim 0.35 \mathrm{au}$ ). A Br $\gamma$ emission dominated by magnetospheric accretion processes would emerge from a radius of about $\sim 0.03 \mathrm{au}$ that is 5 times smaller than what we derived from the measurements. This extended $\mathrm{Br} \gamma$-line emission suggests that the dominant emitting mechanism is more likely a disc wind rather than emission from the magnetosphere. To interpret our AMBER observations, we employed a magnetocentrifugally driven disc-wind model and found that it can approximately reproduce the measured visibilites and phases of MWC 120 (see Fig. 1). For further analysis, we compare our results to other Herbig Ae/Be stars.

In Fig. 3, the inner radius of the disc-wind-launching region of our best model is compared to the inner radii of earlier studies of other Herbig Ae/Be stars (Weigelt et al. 2011; Caratti o Garatti et al. 2015; Garcia Lopez et al. 2015; Kurosawa et al. 2016). Here, we plot the inner radii of the disc-wind-launching regions as well as the $K$-band continuum inner radii as a function of stellar luminosity. Our new MWC 120 results are plotted with solid square symbols. Four stars of the sample, MWC 275, HD 58647, HD 98922 and our new observation of MWC 120 have similar spectral types (A1-B9). The star MWC 297 is of early spectral type (B1.5). This size-luminosity plot shows that the inner radius of the wind-launching region increases with increasing stellar luminosity. We overplot the data points with the power law found earlier $\omega_{1}=2 \times 10^{-3} L_{\star}^{0.6}$ (Kurosawa et al. 2016, dashed black line). For a physical interpretation, we plot the Alfvén radius $r_{A}$ (the radius where the magnetic energy density is equal to the kinetic energy density of the gas) for each target for the stellar parameters in Table 5 using the standard equation (see e.g., Wang 1996):

$$
r_{A} \approx\left(\frac{B_{\star}^{4} R_{\star}^{12}}{2 G M_{\star} \dot{M}_{\mathrm{acc}}^{2}}\right)^{1 / 7},
$$

where $B_{\star}$ denotes the stellar surface magnetic field, $R_{\star}$ the stellar radius, $M_{\star}$ the stellar mass, $\dot{M}_{\text {acc }}$ the accretion rate, and $G$ the gravitational constant. For the whole sample we assume a magnetic field strength on the stellar surface between 25-150 G, reasonable for Herbig Ae/Be stars (see e.g. Wade et al. 2007) indicated by the grey area in Fig. 3. The red squares are the Alfvén radii of our sample for an intermediate value of the surface magnetic field of $75 \mathrm{G}$. The comparison shows that the Alfvén radius can approximately describe the location of the modelled inner disc-wind radius for each target. Only for the high-luminosity object MWC 297, Eqn. 3 predicts a much smaller value. To match the measured radius of around 0.5 au with Eqn. 3, either the mass accretion rate must be very low $\left(\sim 10^{-10} M_{\odot} \mathrm{yr}^{-1}\right)$ or the assumed magnetic field must be of the order of $2 \mathrm{kG}$. While the disc-wind-launching region in the four late spectral type stars is about 10 times smaller than the $K$-band continuum region, they are roughly similar in the
Table 5. Stellar parameters for the Herbig Ae/Be sample

\begin{tabular}{lccccc}
\hline Targetname & $\begin{array}{c}\mathrm{L} \\
{\left[L_{\odot}\right]}\end{array}$ & $\begin{array}{c}R_{\star} \\
{\left[R_{\odot}\right]}\end{array}$ & $\begin{array}{c}M_{\star} \\
{\left[R_{\odot}\right]}\end{array}$ & $\begin{array}{c}\dot{M}_{\text {acc }}{ }^{a} \\
{\left[M_{\odot} \mathrm{yr}^{-1}\right]}\end{array}$ & References \\
\hline MWC 275 & 40 & 2.3 & 2.2 & $0.8 \times 10^{-7}$ & 1 \\
MWC 120 & 100 & 4.6 & 4.3 & $3.5 \times 10^{-7}$ & 2 \\
HD58647 & 412 & 6.2 & 4.6 & - & 3 \\
HD98922 & 640 & 7.6 & 5.2 & $1.07 \times 10^{-7}$ & 4,5 \\
MWC 297 & 10600 & 6.1 & 10 & - & 6 \\
\hline
\end{tabular}

Notes: ${ }^{a}$ If not available from literature, we assumed a mass accretion rate of $10^{-7} M_{\odot} \mathrm{yr}^{-1}$.

References: (1) Garcia Lopez et al. (2015); (2) Alecian et al. (2013); (3) Kurosawa et al. (2016); (4) Caratti o Garatti et al. (2015); (5) Fairlamb et al. (2015); (6) Weigelt et al. (2011)

case of MWC 297 (see Fig. 3, data points on the far right). Possible explanations were already discussed in literature (e.g. Weigelt et al. 2011; Hone et al. 2017, and references therein) and might include the absorption of the stellar light by an inner gas disc allowing dust to survive closer to the star. Although most Herbig Ae/Be stars are expected to host weak magnetic fields, some massive stars may host stronger magnetic fields of a few kG (Wade et al. 2016).

\section{SUMMARY AND CONCLUSION}

We present the first near-infrared interferometric observations resolving the $\mathrm{Br} \gamma$-line emitting region of the Herbig Ae/Be star MWC 120. The following results were obtained.

(i) Geometric modelling of the near-infrared continuum and line visibilites reveals a $\mathrm{Br} \gamma$ line-emitting region $\left(R_{\text {line }} \sim 0.4\right.$ mas corresponding to $\left.0.15 \mathrm{au}\right)$ about two times more compact than then $K$-band continuum $\left(R_{\text {cont }} \sim 0.9\right.$ mas corresponding to $0.34 \mathrm{au}$ ).

(ii) All visibilities and phases of our MWC 120 observations can be approximately reproduced by a state-of-the-art disc-wind model with an inner disc-wind base radius $\omega_{1}$ of $2 R_{\star}(0.04 \mathrm{au})$ and an inner half opening angle of $45^{\circ}$. The orientation of the disc symmetry axis can be approximated by the intrinsic V-band polarization angle (Grinin et al. 1991). The outflow direction (system axis) towards the PA of $135^{\circ}$ used in our models is in agreement with the measured linear polarization angle between $115-138^{\circ}$ (Mottram et al. 2007; Oudmaijer \& Drew 1999; Yudin 2000).

(iii) The derived inner radius of the disc-wind base of the MWC 120 best model is in agreement to the size-luminosity relation for other Herbig Ae/Be stars following a power law distribution of the form $\omega_{1}=2 \times 10^{-3} L_{\star}^{0.6}$ reported earlier.

(iv) For stars of late spectral type (A1-B9), the derived inner radii of the disc-wind base can be approximately described by the corresponding Alfvén radii for each target.

Interferometric observations of the $\mathrm{Br} \gamma$ line-emitting regions in high spectral and high spatial resolution of Herbig Ae/Be stars are of great importance to reveal the dominant physical origin, e.g. the emission emerging from a larger scale 
disc wind or from the more compact magnetosphere near the central star. The presented geometric and radiative transfer modelling of MWC 120 and the other stars discussed above show a trend towards a disc-wind scenario where the observed $\mathrm{Br} \gamma$ line emission is dominantly emerging from a region more extended than the magnetosphere. The inner disc-wind radius seems to be related to the Alfvén radius and might therefore imply an indirect link between the accretion and ejection mechanism in YSOs as already stated by Kraus et al. (2008). More detailed interferometric observations of Herbig Ae/Be stars with the next-generation $K$-band interferometer VLTI/GRAVITY with high spectral and high spatial resolution are required to increase the current statistics on the $\mathrm{Br} \gamma$-emission size distribution. Better statistics together with further measurements of magnetic fields in Herbig Ae/Be stars will allow us to enhance our understanding of the physical mechanism involved in the creation of disc winds and the accretion-ejection mechanism in intermediate mass YSOs.

\section{ACKNOWLEDGEMENTS}

This research has made use of NASA's Astrophysics Data System Bibliographic Services. We thank the ESO staff at Paranal for their support during the observations. We acknowledge support from an STFC Rutherford Fellowship (ST/J004030/1), Rutherford Grant (ST/K003445/1) and ERC Starting Grant (Grant Agreement No. 639889).

\section{REFERENCES}

Alecian E., et al., 2013, MNRAS, 429, 1001

Blandford R. D., Payne D. G., 1982, MNRAS, 199, 883

Caratti o Garatti A., et al., 2015, A\&A, 582, A44

Cauley P. W., Johns-Krull C. M., 2015, ApJ, 810, 5

Eisner J. A., Lane B. F., Hillenbrand L. A., Akeson R. L., Sargent A. I., 2004, ApJ, 613, 1049

Fairlamb J. R., Oudmaijer R. D., Mendigutía I., Ilee J. D., van den Ancker M. E., 2015, MNRAS, 453, 976

Garcia Lopez R., Natta Testi L., Habart E., 2006, A\&A, 459, 837

Garcia Lopez R., Tambovtseva L. V., Schertl D., Grinin V. P., Hofmann K.-H., Weigelt G., Caratti o Garatti A., 2015, A\&A, 576, A84

Garcia P. J. V., Ferreira J., Cabrit S., Binette L., 2001, A\&A, 377,589

Grinin V. P., Katysheva N. A., 1980, Bulletin Crimean Astrophysical Observatory, 62, 52

Grinin V. P., Mitskevich A. S., 1990, Astrophysics, 32, 216

Grinin V. P., Tambovtseva L. V., 2011, Astronomy Reports, 55, 704

Grinin V. P., Kiselev N. N., Chernova G. P., Minikulov N. K., Voshchinnikov N. V., 1991, IAU, 186, 283

Harrington D. M., Kuhn J. R., 2007, ApJ, 667, L89

Hone E., Kraus S., Kreplin A., Hofmann K.-H., Weigelt G., Harries T., Kluska J., 2017, A\&A, 607, A17

Kraus S., et al., 2008, A\&A, 489, 1157

Kreplin A., Kraus S., Hofmann K.-H., Schertl D., Weigelt G., Driebe T., 2012, A\&A, 537, 103

Kurosawa R., et al., 2016, MNRAS, 457, 2236

Kurucz R. L., 1979, ApJS, 40, 1

Lazareff B., et al., 2017, A\&A, 599, A85

Mariñas N., Telesco C. M., Fisher R. S., Packham C., 2011, ApJ, 737,57
Merrill P. W., Burwell C. G., 1933, Contributions from the Mount Wilson Observatory / Carnegie Institution of Washington, 471, 1

Monnier et al., 2005, ApJ, 624, 832

Mottram J. C., Vink J. S., Oudmaijer R. D., Patel M., 2007, MNRAS, 377, 1363

Muzerolle J., Hartmann L., Calvet N., 1998, AJ, 116, 455

Muzerolle J., D'Alessio P., Calvet N., Hartmann L., 2004, ApJ, 617,406

Oudmaijer R. D., Drew J. E., 1999, MNRAS, 305, 166

Petrov R. G., et al., 2007, A\&A, 464, 1

Safier P. N., 1993, ApJ, 408, 115

Sobolev V. V., 1960, Soviet Ast., 4, 372

Tambovtseva L. V., Grinin V. P., Rodgers B., Kozlova O. V., 2001, Astronomy Reports, 45, 442

Tambovtseva L. V., Grinin V. P., Weigelt G., 2014, A\&A, 562, A104

Tambovtseva L. V., Grinin V. P., Weigelt G., 2016, A\&A, 590, A97

Tatulli E., et al., 2007, A\&A, 464, 29

Vinković D., Jurkić T., 2007, ApJ, 658, 462

Wade G. A., Bagnulo S., Drouin D., Landstreet J. D., Monin D., 2007, MNRAS, 376, 1145

Wade G. A., et al., 2016, MNRAS, 456, 2

Wang Y.-M., 1996, ApJ, 465, L111

Weigelt G., et al., 2011, A\&A, 527, 103

Yudin R. V., 2000, Astronomy and Astrophysics Supplement, 144, 285

de Winter D., van den Ancker M. E., Maira A., The P. S., Djie H. R. E. T. A., Redondo I., Eiroa C., Molster F. J., 2001, A\&A, 380,609

van den Ancker M. E., 2005, Tracing Accretion onto Herbig Ae/Be Stars Using Near-Infrared Spectroscopy. Springer Berlin Heidelberg, Berlin, Heidelberg, pp 309314, doi:10.1007/10995082_48, https://doi.org/10.1007/ 10995082_48

van den Ancker M. E., de Winter D., Tjin A Djie H. R. E., 1998, A\&A, 330, 145

\section{APPENDIX A: GEOMETRIC MODEL FIT}

This paper has been typeset from a $\mathrm{T}_{\mathrm{E}} \mathrm{X} / \mathrm{LAT}_{\mathrm{E}} \mathrm{X}$ file prepared by the author. 


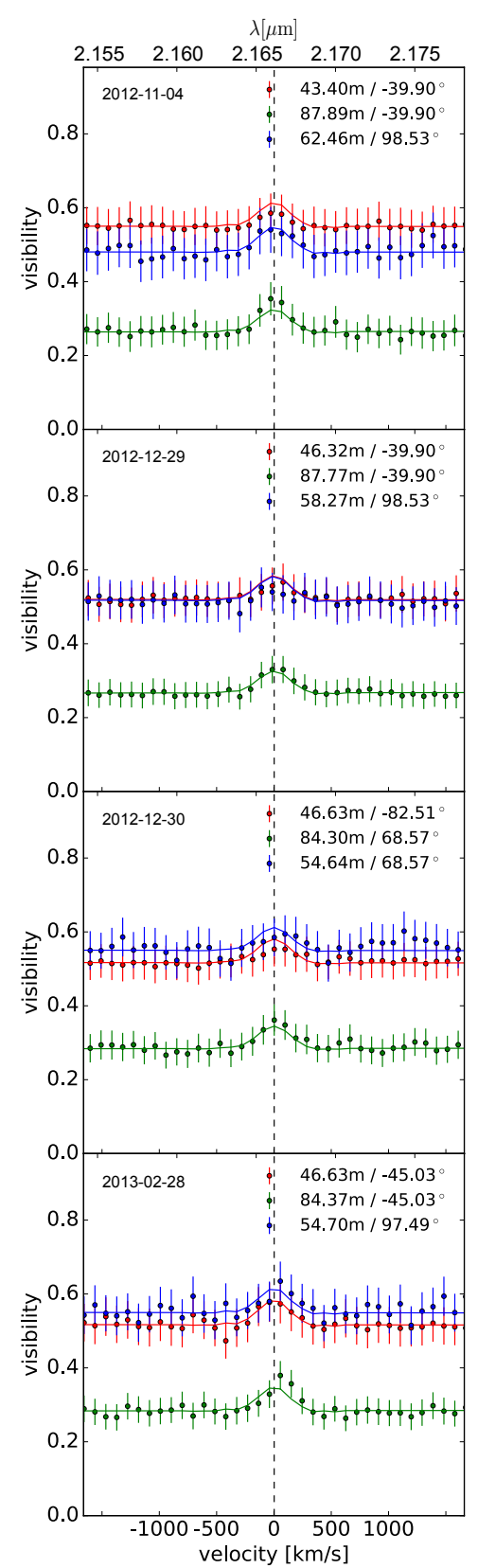

Figure A1. Best fit of the continuum and line visibilities of MWC 120 (different data sets from top to bottom) assuming a temperature-gradient model for the continuum emission and $\mathrm{Br} \gamma$-line emission (Model A; see best-fitting parameters in table 2 ). The data points show the observations for different baseline lengths (red, green, blue). The best-fitting model is plotted as solid lines. The $2.166 \mu \mathrm{m} \mathrm{Br} \gamma$-line position is indicated with a dashed line. 